\title{
Imaging of the Postoperative Anterior Cruciate Ligament: Emphasis on New Surgical and Imaging Methods
}

\author{
Pieter Van Dyck, MD, PhD ${ }^{1}$ Valérie Lambrecht, MD $\quad$ Eline De Smet, MD ${ }^{1}$ Anagha P. Parkar, MD ${ }^{3,4}$ \\ Christiaan H. W. Heusdens, MD ${ }^{5}$ Martijn F. Boomsma, MD ${ }^{6}$ Filip M. Vanhoenacker, MD, PhD ${ }^{1,2,7}$ \\ Jan L. Gielen, MD, $\mathrm{PhD}^{1}$ Paul M. Parizel, MD, $\mathrm{PhD}^{1}$
}

${ }^{1}$ Department of Radiology, Antwerp University Hospital and University of Antwerp, Edegem, Belgium

2 Department of Radiology, Ghent University Hospital, Ghent, Belgium

${ }^{3}$ Department of Radiology, Haraldsplass Deaconess Hospital, Bergen, Norway

${ }^{4}$ Department of Clinical Medicine, Faculty of Medicine and Dentistry, University of Bergen, Bergen, Norway

${ }^{5}$ Department of Orthopaedics, Antwerp University Hospital and University of Antwerp, Edegem, Belgium

${ }^{6}$ Department of Radiology, Isala Hospital, Zwolle, the Netherlands

7 Department of Radiology, AZ Sint-Maarten, Duffel, Belgium
Address for correspondence Pieter Van Dyck, MD, PhD, Department of Radiology, Antwerp University Hospital and University of Antwerp Wilrijkstaat 10, 2650 Edegem, Belgium

(e-mail: pieter.van.dyck@uza.be).

Semin Musculoskelet Radiol 2016;20:33-42.

\begin{abstract}
Keywords

- anterior cruciate ligament ( $\mathrm{ACL})$ reconstruction

- ACL repair

- magnetic resonance imaging (MRI)

- quantitative MRI

The aim of anterior cruciate ligament $(\mathrm{ACL})$ reconstruction is to restore normal function of the knee, but unfortunately abnormal kinematics and a predisposition to knee osteoarthritis occur in a significant percentage of patients. So there is an ongoing need to improve treatment options and long-term outcome of patients with a ruptured ACL. With the recent advancements in the field of $A C L$ tissue engineering, the focus of treatment for $A C L$ injuries is changing from resection and reconstruction toward repair and regeneration. Several new $A C L$ repair methods were recently introduced as an alternative to traditional $\mathrm{ACL}$ reconstructive procedures. Radiologists must become familiar with these new surgical methods to interpret their appearance correctly on postoperative imaging studies. This article provides an overview of the latest advancements in ACL surgical methods and discusses the role of imaging to assess the postoperative $A C L$ including both standard and advanced imaging methods.
\end{abstract}

One of the most challenging problems facing orthopedics today is the failure of tissues within the joint, such as the anterior cruciate ligament $(\mathrm{ACL})$, to heal naturally after injury and surgical repair. ${ }^{1,2}$ Therefore, surgical resection and reconstruction of the ACL is often recommended to facilitate a return to desired daily activities including sports. However, an incidence of osteoarthritis (OA) as high as $50 \%$ within 7 to 14 years after injury and reconstruction of the ACL is still a main drawback of this surgical strategy. ${ }^{3}$ In contrast, a successful ACL repair can theoretically provide the patient with multiple advantages over surgical reconstruction, including preservation of the proprioceptive function of the ligament and the complex ligament insertion sites. ${ }^{2}$

Research in the field of ACL tissue engineering is ongoing and on the rise. Recently, several novel ACL repair techniques showed satisfactory outcomes in preclinical studies. ${ }^{4,5}$ The potential success of these new ligament-preserving techniques may result in a realistic alternative for ACL reconstruction.

This article provides an overview of the latest advancements in ACL surgical methods and discusses the role of imaging for the assessment of the postoperative ACL, including recent developments in imaging and the new insights they may provide.
Issue Theme Knee and Ankle Imaging; Guest Editor, Marco Zanetti, MD
Copyright @ $₫ 2016$ by Thieme Medical Publishers, Inc., 333 Seventh Avenue, New York, NY 10001, USA. Tel: +1(212) 584-4662.
DOI http://dx.doi.org/ 10.1055/s-0036-1579678. ISSN 1089-7860. 


\section{ACL Injury and Repair}

\section{Native ACL Healing}

In humans, the intra-articular ACL fails to heal after complete rupture. $^{2}$ This makes the ACL vastly different from other extra-articular ligaments in the body including the medial collateral ligament (MCL), which heals readily with functional bracing. ${ }^{4}$ Initially, it was suggested that the lack of healing was due to the failure of the cells and blood vessels within and around the ACL to mount an adequate response. ${ }^{4}$ Recent work found that the human ACL has a proliferative vascular and neurogenic response to rupture, a response similar to that found in the injured MCL. ${ }^{2,4,5}$ In addition, there was no bridging of the gap between the ruptured ends of the ACL. Most connective tissues heal by filling the wound gap with a fibrin-platelet clot or provisional scaffold. The ACL wound site is not bridged by clot or connective tissue. Thus it was hypothesized that the failure of the ACL to heal was a result of a lack of wound-site filling. ${ }^{2,4,5}$ The persistence of an unfilled gap in the wound site is also observed in meniscus, cartilage, and rotator cuff tendons, and all tissues within the intra-articular environment.

Several studies suggest that increased levels of so-called clot-busting enzymes that appear in the joint fluid shortly after trauma may prevent formation of a blood clot. This is a natural mechanism probably minimizing joint fibrosis after an injury, thus preserving motion within the joint and maintaining short-term function at the expense of tissue healing and long-term joint function. ${ }^{2,4}$ The hypothesis is that filling the wound site in vivo with an appropriate substitute for a clot may lead to improved healing. ${ }^{4,5}$ The ideal substitute scaffold has to be biocompatible and biodegradable to enable tissue ingrowth, and its mechanical properties should mimic the natural ACL as closely as possible. This is crucial for the native ligament to heal. ${ }^{5}$

\section{ACL Graft Healing}

Graft healing after ACL reconstruction occurs at two different sites: intra-articular graft remodeling, often referred to as ligamentization, and intratunnel graft incorporation. ${ }^{6,7}$ Previous studies have demonstrated three characteristic stages of intra-articular graft healing after ACL reconstruction: an early healing phase with central graft necrosis and hypocellularity and no detectable graft revascularization of the graft tissue, followed by a phase of proliferation, the time of most intensive remodeling and revascularization, and finally, a ligamentization phase, with characteristic restructuring of the graft toward the properties of the native ACL. ${ }^{2,6,7}$ However, full restoration of either the biological or biomechanical properties of the native ACL is never achieved. ${ }^{6}$ In addition, successful ACL reconstruction requires solid bone tunnel healing. The mechanism by which graft-bone healing occurs depends on the type of the graft used. ${ }^{6}$ For bone-patellar tendon-bone grafts, healing in the tunnel resembles normal fracture healing, with incorporation of the bone block in the tunnel observed as early as 16 weeks after surgery. ${ }^{2,6,8}$

The healing process occurs through a different mechanism after implantation of a soft tissue graft without bone plugs.
First, fibrovascular interface tissue forms between graft and bone. Then progressive mineralization of the interface tissue occurs with subsequent bone ingrowth into the outer tendon and incorporation of the tendon graft into the surrounding bone. Progressive reestablishment of the continuity of the collagen fibers between the tendon and the bone results in a fibrous insertion of the tendon., ${ }^{4}$ Complete bone tunnel healing of the ACL graft may occur as late as 6 to 12 months after surgery. ${ }^{6,8}$ Several methods are used to improve bone tunnel healing, including both mechanical ways (e.g., lengthening the tunnel, minimizing the mismatch of graft-tunnel diameter, and providing circumferential contact between the graft and tunnel) and biological ways (e.g., use of osteoinductive cytokines such as bone morphogenetic proteins). ${ }^{4,6,8}$ Unfortunately, a common cause for failed ACL reconstruction is still a failure of graft-to-bone healing., 6,8

\section{Surgical Treatment of ACL Injury}

\section{Historical Overview and Current Gold Standard}

ACL surgery has evolved considerably over the past decades. The awareness of the inadequacy of direct suture repair (years 1895-1970) and nonanatomical ACL reconstruction techniques (extra-articular lateral tenodesis, late 1970s) led to an almost universal adoption of intra-articular ACL reconstruction (1980-today) for treatment of ACL injuries. ${ }^{9,10} \mathrm{ACL}$ reconstructions are most often performed with hamstring and bone-patella tendon-bone autograft tendons (-Fig. 1). The initial literature proposed bone tunnel placement for best graft isometry (full range of motion without causing ligament elongation and plastic deformation), but there is now compelling evidence to support anatomical tunnel placement (anatomical ACL reconstruction). ${ }^{6,8}$ The transition from a

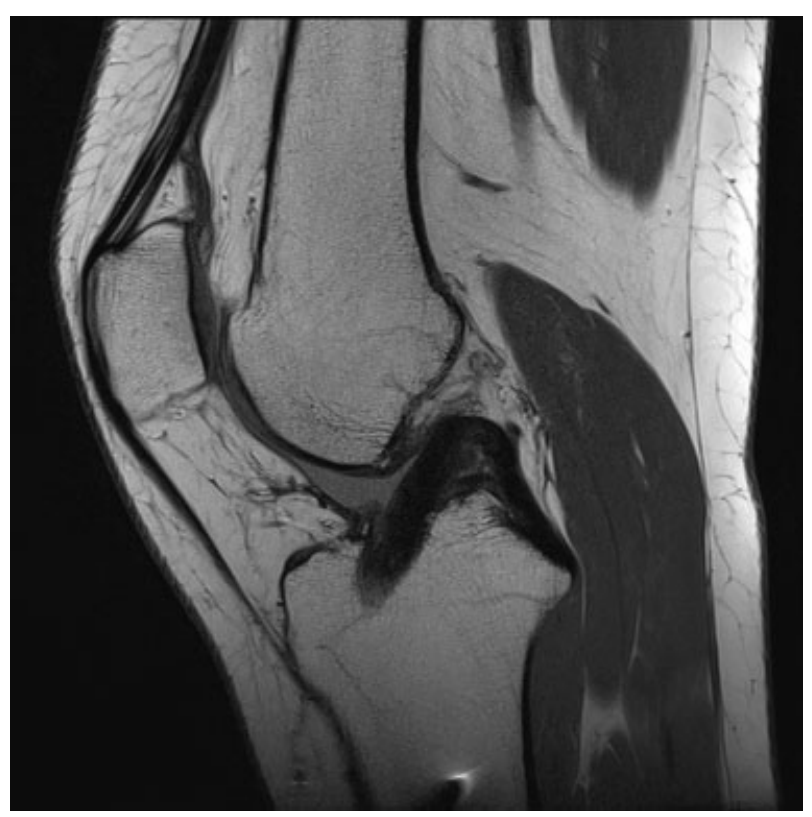

Fig. 1 Anterior cruciate ligament $(A C L)$ reconstruction using hamstring autograft tendon. MRI was performed 14 months after surgery for recurrent knee pain. Sagittal proton-density-weighted image shows homogeneous low signal intensity of the healed ACL graft. 
single-bundle to a double-bundle technique was recommended to reconstruct the anteromedial (AM) and posterolateral (PL) bundles separately to imitate the normal anatomy of the native $\mathrm{ACL}$, thus helping to restore knee stability more effectively. ${ }^{6,8}$ As of late, there have been reports of no difference in outcome measures between the two techniques, particularly when the reconstruction is anatomical. $6,8,11$

To improve outcomes, further modifications have been introduced to the ACL reconstruction technique such as selective (AM or PL) bundle augmentation in partial ACL tears, and, more recently, reconstruction with preservation of remnant ligament tissue. ${ }^{6,8,11}$ Preservation of ACL remnants seems theoretically beneficial in terms of vascularity, proprioception, and kinematics. However, none of these modifications have been shown to make a significant difference to patient-reported outcomes. Moreover, retention of the ACL remnant tissue might lead to incorrect tunnel placement and cyclops formation. ${ }^{6,8}$

\section{New Surgical Techniques}

Several methods of primary ACL repair have recently been introduced including dynamic intraligamentary stabilization (DIS), ${ }^{12}$ internal brace ligament augmentation (IBLA), ${ }^{13}$ and primary $\mathrm{ACL}$ reconstruction using a degradable poly L-lactic acid (PLLA) scaffold. ${ }^{14}$

Generally, ACL repair needs to be performed relatively quickly after the initial injury because sufficient viable native ligament tissue must be present. Depending on the technique used, time to surgery after the rupture has to be $<3,12$, or 18 weeks. In contrast, ACL reconstruction is performed from the moment the knee function has recovered, mostly from 3 to 6 weeks, up to any time after the injury.

DIS (Ligamys, Mathys AG, Bettlach, Switzerland) has been introduced for the repair of acute (time to surgery $\leq 3$ weeks after injury) proximal or central ACL tears. ${ }^{12}$ After microfracturing at the femoral footprint, the tibial remnants of the torn ACL are reduced to the femoral footprint by transosseous sutures. The knee is then stabilized with a strong polyethylene cord, which is anchored to the bone much like a standard ACL graft implant on the femoral side with a titanium flip anchor. The cord is pulled through the femur and the anatomical femoral footprint and passed behind the tibial footprint to prevent additional damage to the tibial ACL blood and nerve supply. It is brought under tension by a steel/Phynox screw-spring implant, which is placed at the anteromedial tibia (-Fig. 2). This mechanism acts as a dynamic internal fixator. It pushes the proximal tibia in a constant posterior drawer position ( 50 to $80 \mathrm{~N}$ ) in any degree of flexion, ensuring that the two ligament stumps are kept as close to each other as possible at all times to enable mechanically stable $\mathrm{ACL}$ healing. ${ }^{15}$ The presence of the metallic implant in the tibia after DIS may complicate MRI follow-up in these patients and usually necessitates the use of metal artifact reduction sequences (e.g., slice encoding for metal artifact reduction ${ }^{16}$ ).

The efficacy of DIS in facilitating the self-healing of the ACL was analyzed in a sheep model, and complete healing of the torn ligaments was demonstrated through histologic examination. ${ }^{17}$ Recently, clinical experience of the first 3 years after DIS in a large case series was reported. ${ }^{18}$ The authors concluded that anatomical repositioning, along with DIS and microfracturing, leads to clinically stable healing of the torn ACL in most patients (96\%). Most patients exhibited a normal knee function, reported excellent satisfaction, and were able to return to their previous levels of sporting activity. ${ }^{18}$ However, longer term and comparative follow-up studies are required to determine whether the DIS technique improves clinical outcomes.

The IBLA (Arthrex, Naples, FL, USA) is a ligament repair bridging concept using braided ultrahigh-molecular-weight polyethylene/polyester suture tape and knotless bone anchors to reinforce ligament strength as a secondary stabilizer after repair and return to sports, which may help resist injury recurrence. ${ }^{13}$ This technique involves repair of the ACL (time to surgery $\leq 12$ weeks after injury) where it has ruptured close to its femoral attachment.

First, the torn ligament is reapproximated anatomically against the lateral femoral condyle, freshened with a microfracture probe. Second, the repair is protected by a 2-mm polyethylene tape through arthroscopically drilled femoral
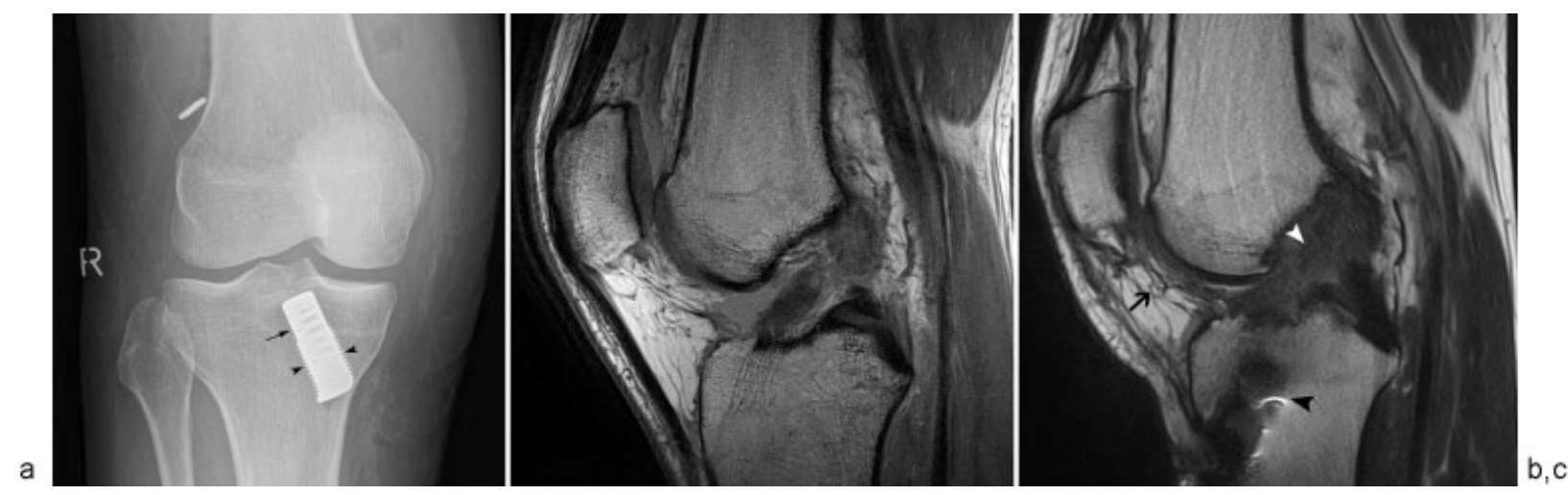

Fig. 2 Dynamic intraligamentary stabilization of the anterior cruciate ligament (ACL). (a) Anteroposterior radiograph demonstrating spring (arrow) screw (arrowhead) mechanism at the anteromedial tibia. Note titanium flip anchor at the lateral femoral cortex. (b) Preoperative sagittal proton-density-weighted image showing acute proximal tear of the ACL and large joint effusion. (c) Follow-up MRI at 6 months after surgery demonstrating clear continuity of the repaired ACL (white arrowhead). Note postoperative scar at Hoffa fat pad (arrow). Only minimal artifacts are seen at the tibia (black arrowhead) with use of slice encoding for metal artifact reduction. 

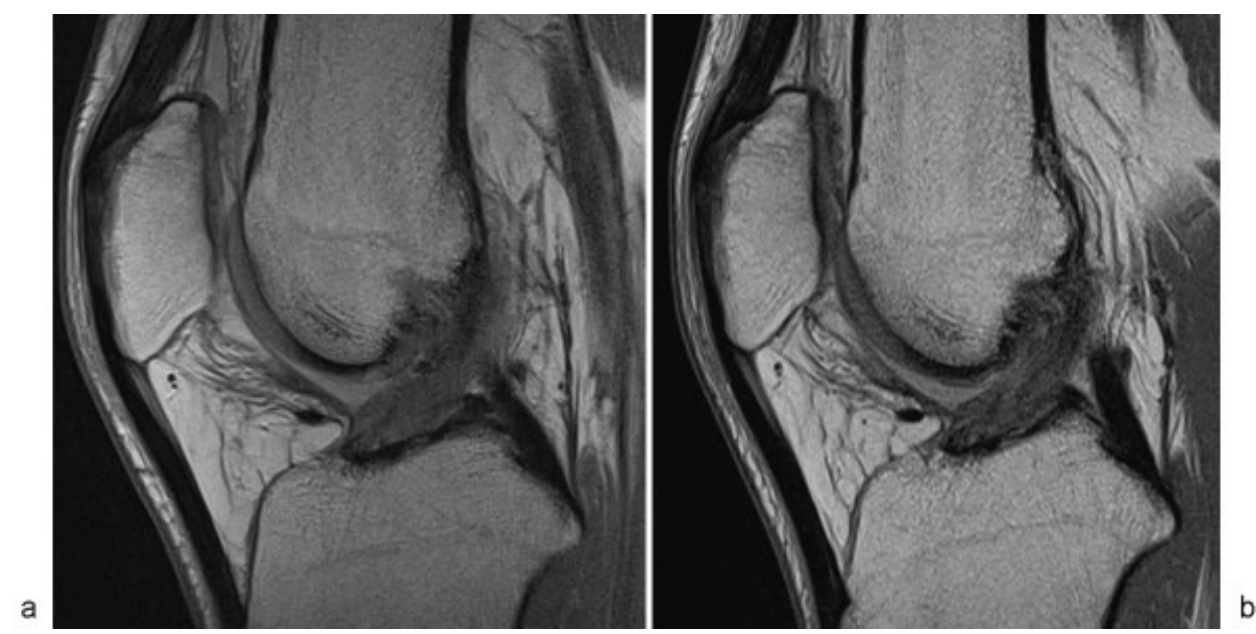

Fig. 3 Internal bracing of the anterior cruciate ligament (ACL). (a) Postoperative sagittal proton-density-weighted image at 5 months. (b) Followup MRI at 12 months showing decrease of the signal intensity of the ACL repair tissue, indicating tissue maturation.

and tibial bone tunnels $(3.5 \mathrm{~mm})$, bridging the anatomical attachments of the ACL in the mid-bundle position from tibia to femur. The tape is fixed on the femur with a titanium or steel flip anchor and on the tibia with a titanium bone anchor. The internal brace supports early mobilization of the repaired ligament and allows the natural tissues to strengthen progressively, protecting it against high strains that would impair the healing or even cause a rerupture during the healing process of the ligament ${ }^{13}$ (-Fig. 3 ). The results of a recent study suggest that at minimum 1-year follow-up, IBLA is at least as effective in restoring stability and function of the knee as traditional ACL reconstruction surgery, with the greatest improvements seen in return to sporting activity. ${ }^{19}$ Longer term follow-up and randomized studies are further required to compare IBLA directly against standard ACL reconstruction techniques.

Primary ACL reconstruction with resorbable PLLA scaffold (L-C Ligament; Soft Tissue Regeneration, Inc., New Haven, CT, USA) is intended for primary ACL reconstruction within 18 weeks of acute ACL rupture. It is a synthetic bioresorbable, three-dimensional (3D) braided polymer scaffold made from PLLA within and around which the native ACL will regenerate over time. ${ }^{14}$ The L-C Ligament is composed of two tightly braided outer segments anchored in the bone tunnels $(8 \mathrm{~mm})$ much like standard ACL graft implants, and a more loosely braided central, intraarticular portion that extends into the apertures of the bone tunnels. The native (ruptured) ACL is left intact as much as reasonably possible with only minimal debridement of ACL stumps. In the short term, the scaffold is strong enough at the time of implantation to allow early functional rehabilitation of the knee. The PLLA scaffold is designed to capture migrating cells and slowly bioresorb over the postoperative period in parallel with neovascularization and native tissue ingrowth. This regenerative process eventually replaces the scaffold with native tissue (-Fig. 4). As resorption occurs, load-bearing responsibility transfers to the tissue ingrowth while mechanical integrity at the site is maintained by the resorbable polymer scaffold. Long-term in vivo performance and biomechanical properties of the L-C Ligament were evaluated in a sheep model, and healing of the torn ligaments with healthy regenerated ACL tissue and (near) complete resorption of PLLA was observed at 12 months, and no adverse effects noted through 4 years. ${ }^{14}$ Further clinical studies are planned to compare ACL reconstruction with PLLA scaffold against autograft surgery with hamstrings.

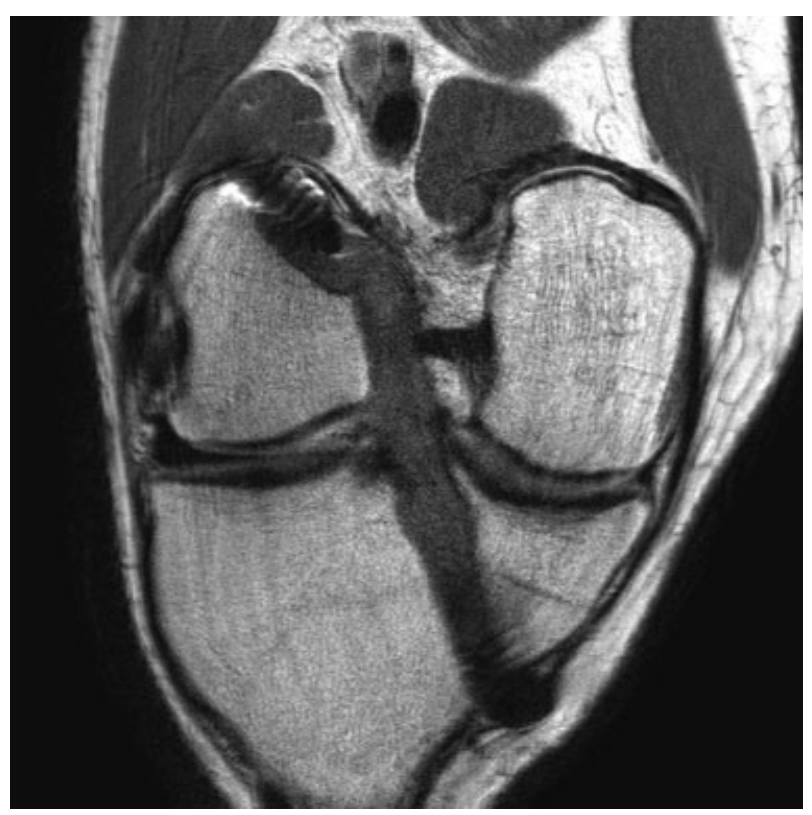

Fig. 4 Primary anterior cruciate ligament $(A C L)$ reconstruction with poly L-lactic acid scaffold. Postoperative paracoronal proton-densityweighted image at 12 months shows intermediate to high signal intensity of the ACL regenerated tissue in a clinically stable knee. (Image courtesy of Soft Tissue Regeneration, Inc., New Haven, CT; Dr. Kees van Egmond and Dr. Martijn Boomsma, Isala Clinics, Zwolle, the Netherlands.) 


\section{Imaging Techniques}

\section{Plain Radiography and Volumetric CT Scan}

Although plain radiography is often the first imaging modality performed after ACL reconstruction (e.g., to assess hardware failure or dislodgment), a computed tomography (CT) scan is more reliable for evaluating the bone tunnels. ${ }^{20}$ Highresolution volumetric $\mathrm{CT}$ acquisition with volume-rendering reconstructions has been used to describe critical bony landmarks of the ACL anatomical footprints that can aid in anatomical ACL reconstruction ${ }^{21}$ (-Fig. 5). This method allows a view from a perspective similar to what is seen at the time of surgery. A grid orientation system can be used to assess femoral and tibial bone tunnel positioning. Commonly used terminology for femoral tunnel placement is shallow/ deep and high/low because surgery is performed with the knee in flexion. The reported optimal placement for deep/ shallow direction is a ratio of $27 \%$, and $34 \%$ in the high/low direction, which corresponds to the anatomical femoral footprint. ${ }^{22,23}$ The reported anatomical central tibial footprint position is located $39 \%$ from anterior and $48 \%$ from medial on the grid system. ${ }^{24}$ Traditionally, the tibial tunnel has been placed more posteriorly in the footprint in an effort to avoid roof impingement. However, the most recent surgical trend is to aim for an anatomical central tibial tunnel location. ${ }^{6,8,24}$ Bone tunnel orientation may vary according to the used drilling technique. ${ }^{6,8,11}$ Transtibial tunnel drilling often results in nonanatomical placement of the femoral tunnel located high in the femoral notch $(<39$ degrees to line parallel to the femoral shaft). ${ }^{11}$ This increases the risk of vertical positioning in the intercondylar notch and positioning anterior to the native $\mathrm{ACL}$ attachment. The anteromedial portal drilling creates a femoral tunnel independent from the tibial tunnel, allowing for a more oblique positioning of the graft and for a tunnel that more closely resembles the native anatomical footprint. ${ }^{8,11}$

CT scan is the best modality for evaluating bone tunnel widening and the first choice modality in the management of
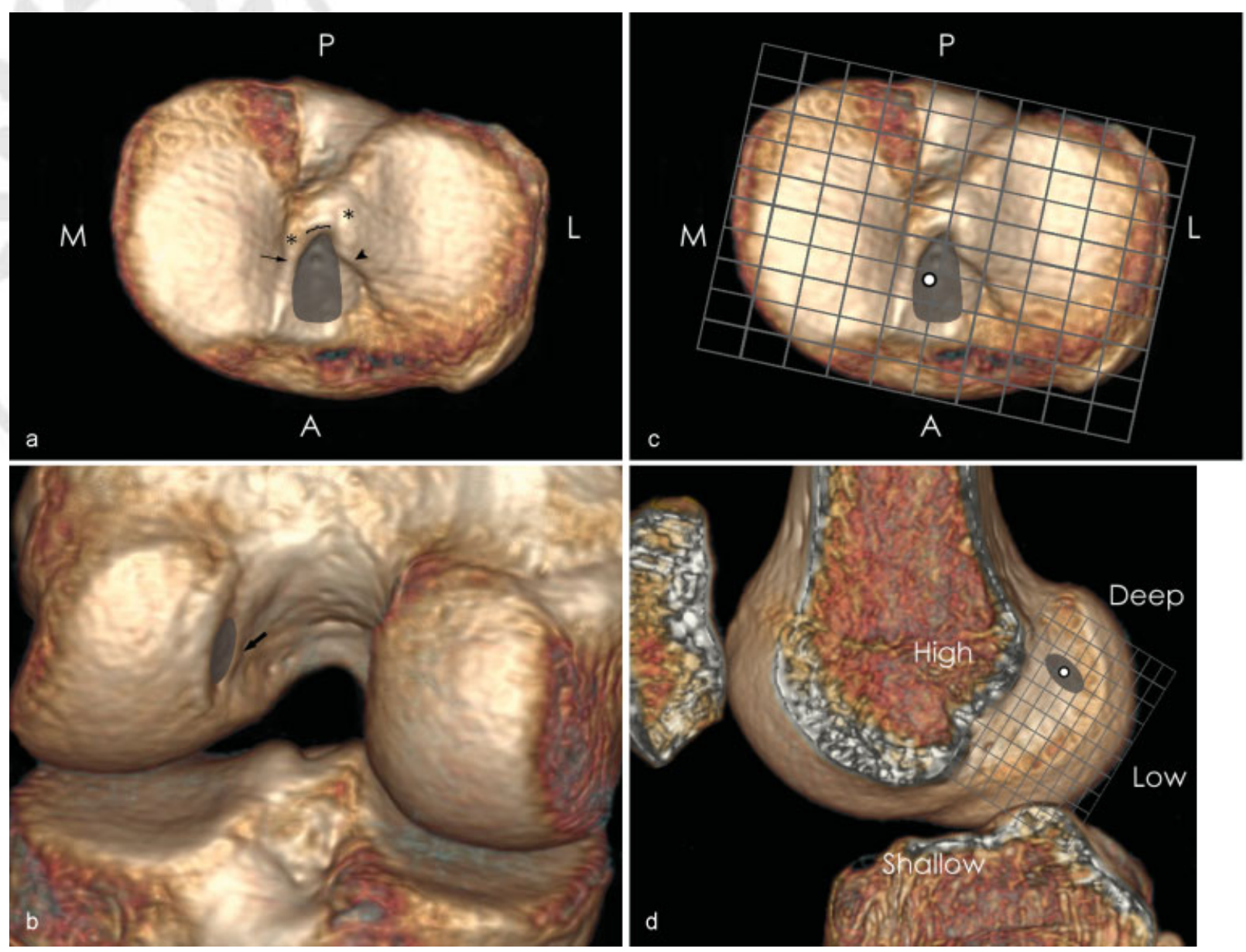

Fig. 5 Computed tomography (CT) scan with volume-rendering reconstructions showing anterior cruciate ligament (ACL) attachments (gray area). (a) Superior view of the left tibia. ACL fibers extend up to the base of the tibial spine, but none of them insert on the tibial spine. Posterior fibers of the $\mathrm{ACL}$ are seen just anterior to a bony ridge that runs between the medial and lateral tibial intercondylar tubercles (asterisks), called the tibial ACL ridge (curly bracket). Medially, ACL fibers insert directly onto the anterior extension of the medial intercondylar tubercle, the medial intercondylar ridge (arrow), near the lateral border of the articular surface of the medial tibial plateau. There is no distinct bony lateral or anterior border of the ACL tibial footprint (lateral intercondylar ridge, arrowhead). (b) Posterior view of the left knee showing femoral ACL attachment. Note bony ridge at the anterior margin of the femoral footprint, called resident's ridge (arrow). Grid orientation system at the (c) tibial and (d) femoral ACL attachment. The reported optimal placement of bone tunnels is marked by a white dot in the footprint (see text for details). 
ACL revision surgery to assess bone stock quality and bone graft incorporation. ${ }^{20,25}$ The radiolucency around the graft associated with tunnel widening is the apparent result of bone resorption in the insertion site area. ${ }^{25,26}$ This finding is supported by MRI data, showing fluid signal, rather than bone signal, surrounding the tendon. The presence of bone resorption at the graft insertion site as early as 3 months after ACL reconstruction needs to be followed closely.

\section{Conventional MR Imaging}

MRI is commonly used as a clinical tool for qualitatively monitoring ACL graft status after surgical reconstruction. ${ }^{27}$ The healed ACL graft has low signal on conventional MRI (-Fig. 1). Intermediate signal may be seen within grafts from $\sim 4$ to 8 months after surgery due to graft remodeling, decreasing with time and usually completely resolving by 12 months. $^{7,27}$ Similarly, bone tunnel healing may be observed on MRI with high signal intensity at the initial bone-tendon interface gradually changing to low signal intensity due to progressive maturation and ossification of the fibrous tissues. ${ }^{26}$

Although human biopsy studies have shown that neovascularization of a tendon autograft occurs, the extent of vascularity is below the threshold detectable with gadolinium-enhanced MRI using conventional sequences. ${ }^{7,28}$ On the contrary, enhancement of the richly vascularized periligamentous soft tissues can be seen on conventional MRI. ${ }^{28}$

In the setting of a reinjury or a poorly functioning graft, the reconstructed ACL is difficult to assess on MRI. A study by Waltz et $\mathrm{al}^{29}$ in 2014 found a $60 \%$ sensitivity and a $87 \%$ specificity of MRI to detect an ACL graft tear. Moreover, the status of the ACL graft on MRI may not correlate well with the actual function of the graft. ${ }^{29-31}$

Preoperative MRI must be performed relatively quickly after the initial injury in patients for whom ACL repair is considered. In particular, the DIS technique is suited for ACL ruptures not older than 2 or 3 weeks. Importantly, radiologists should describe ACL rupture location (proximal, central or distal third) and status of the ligament stumps (single strand, two bundles, three or more strands) because this technique is intended for proximal or central ACL ruptures. ${ }^{18}$

MRI also documents the healing process after primary ACL repair. The signal intensity of the repaired ACL depends on the integrity of the scaffold, the extent of tissue ingrowth, and its degree of maturation (-Fig. 3). The PLLA scaffold gives a uniform low signal on MRI when freshly placed. This low signal is slowly lost by the progressive regeneration and ingrowth of native ACL tissue. As the PLLA continues to be degraded over the postoperative period, the regenerated ACL may still display intermediate or high signal intensity on MRI, even in a clinically stable knee ( - Fig. 4). Dynamic contrastenhanced MRI may be useful to monitor ACL healing and help differentiate between ACL repair tissue and postoperative scar (-Fig. 6).

\section{Advanced MR Imaging}

MR parameters of volume (a measure of tissue quantity) and signal intensity (a surrogate measure of tissue quality) have
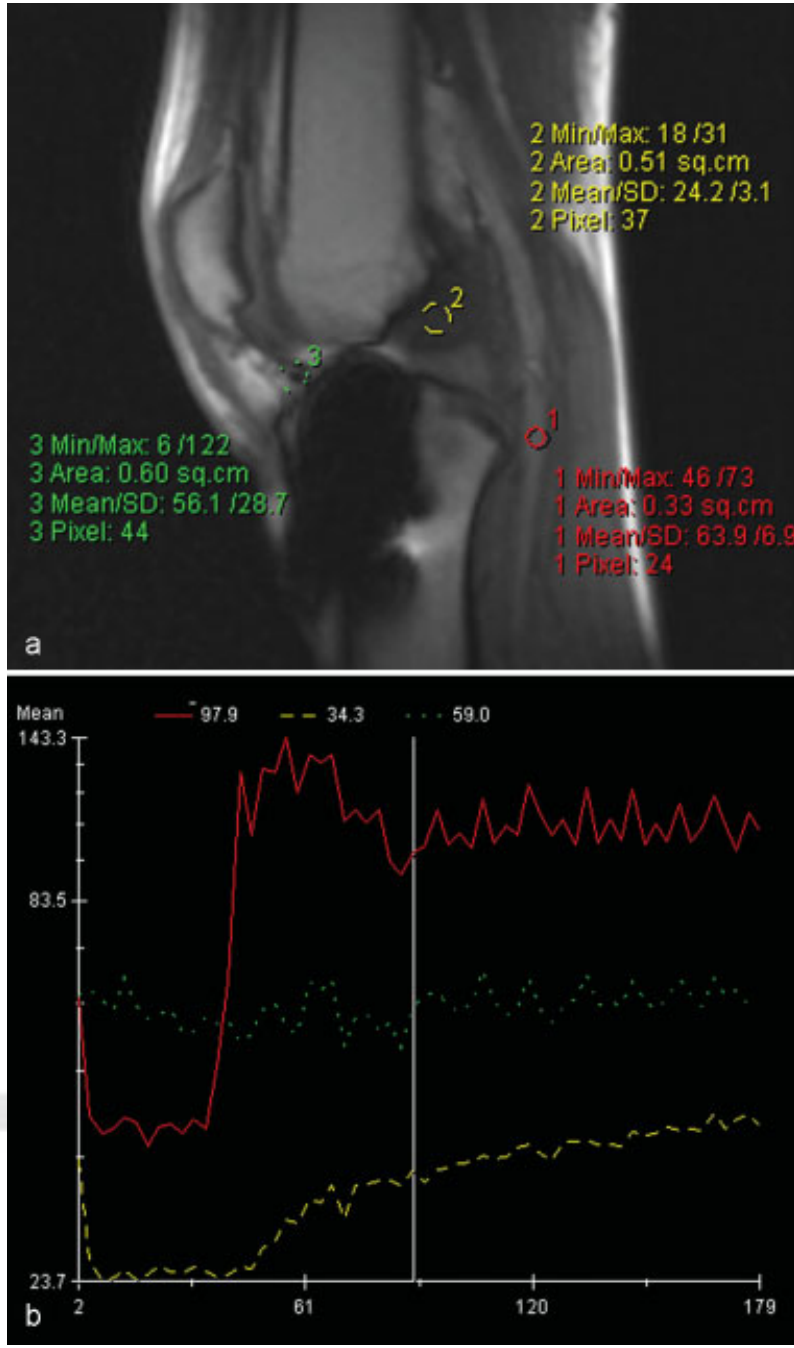

Fig. 6 Dynamic contrast-enhanced MRI after dynamic intraligamentary stabilization of the anterior cruciate ligament (ACL). (a) Region of interest measurements in ACL repair tissue (yellow), Hoffa scar (green), and popliteal artery (red). (b) Dynamic contrast curve shows marked hypervascularity of ACL repair tissue compared with lower vascularity in the postoperative scar.

been found to correlate with the biomechanical properties of an ACL graft and can predict clinical, functional, and patientoriented outcome measures after ACL reconstruction. ${ }^{31,32}$ Because the use of signal intensity as an outcome measure is limited by its dependence on image acquisition parameters and scanner manufacturer, $\mathrm{T} 2$ and $\mathrm{T} 2{ }^{*}$ relaxation time variables are used to standardize MR results $\mathbf{s}^{31,33}$ (-Fig. 7). These time variables are inherent tissue properties that reflect specific tissue characteristics and correlate well with the level of tissue organization. ${ }^{31-33}$ Future work will be needed to validate longitudinally this MRI-based prediction method to document within-patient temporal changes relating to ACL strength and to determine the appropriate timing for athletes to return to sports. ${ }^{32}$

Diffusion tensor imaging (DTI) can be used to image and visualize the structure of the ACL and ACL graft, and it can provide additional information over conventional MRI. ${ }^{34}$ It is a technique particularly suited for the evaluation of highly 

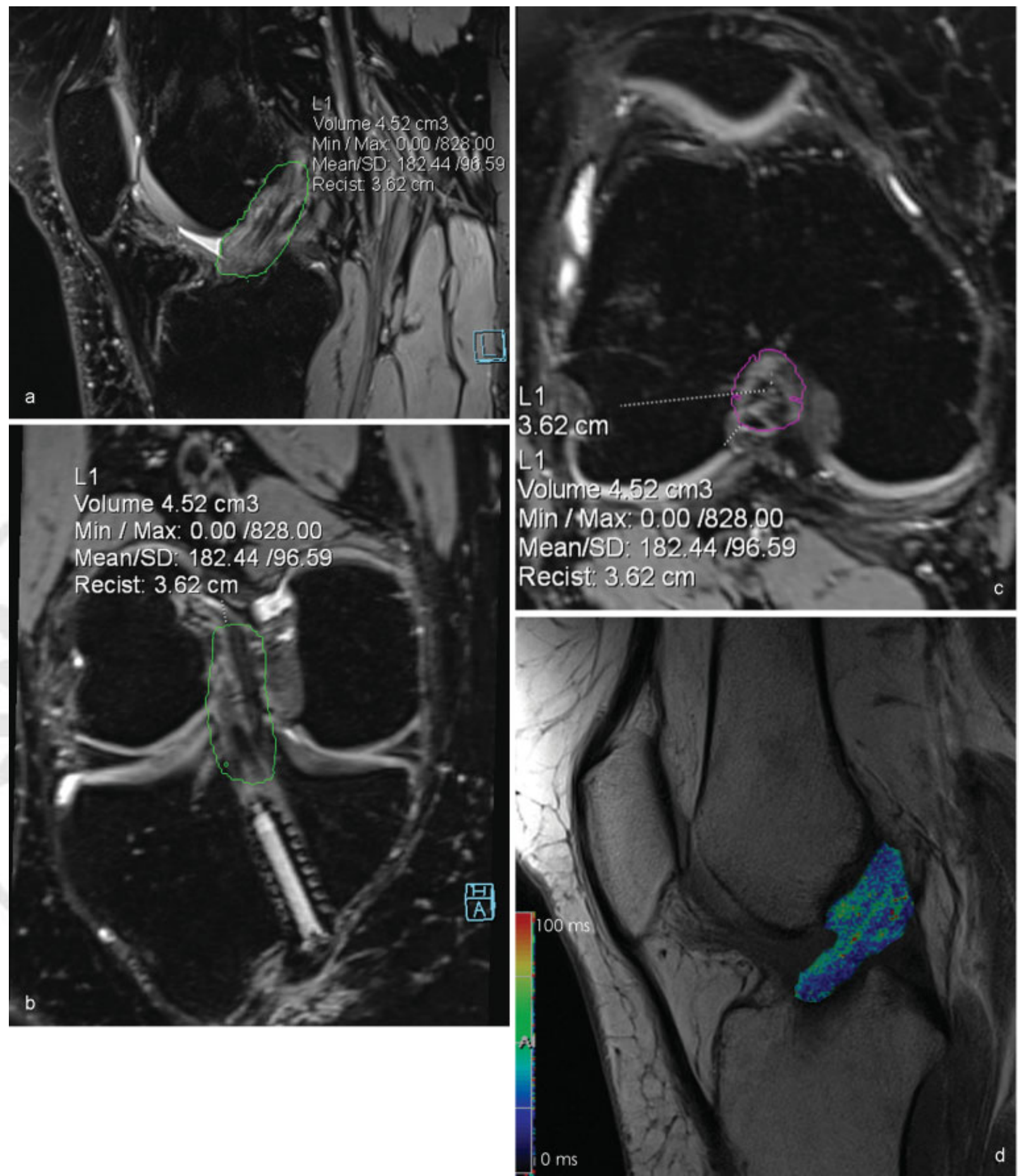

Fig. 7 MRI-based volume measurements and quantitative T2 mapping of anterior cruciate ligament (ACL) graft in clinically stable knee. (a) Sagittal, (b) coronal, and (c) axial three-dimensional fs turbo spin-echo images showing volume measurements of intra-articular ACL graft. (d) Quantitative T2 mapping.

organized tissues and provides quantitative parameters of the fiber bundles, such as fractional anisotropy and apparent diffusion coefficient values. Although ligament DTI is technically challenging because of the small volume of tissue, previous studies showed the feasibility of DTI of the ACL graft. ${ }^{34,35}$ In the author's institution, DTI of the knee is performed on a clinical 3-T MR imaging system (Magnetom PRISMA $^{\text {fit }}$, Siemens AG, Erlangen, Germany) using a 15-channel knee coil and a spin-echo-based echo-planar imaging sequence (336 axial slices; voxel size $1.5 \mathrm{~mm} \times 1.5 \mathrm{~mm} \times 6$ $\mathrm{mm}$; field of view $192 \mathrm{~mm} \times 192 \mathrm{~mm}$; TR/TE 1300/45 msec; b-values 0 and $400 \mathrm{~s} / \mathrm{mm}^{2}$; and acquisition time, 7 minutes, 25 seconds) (-Fig. 8). Further studies are needed to investigate the ability of DTI to monitor quantitatively the healing ACL and to detect ACL graft pathology.

Ultrashort echo time imaging (UTE) is generally applied to techniques using shorter radiofrequency excitation pulses and faster readout methods than conventional methods to produce 
a
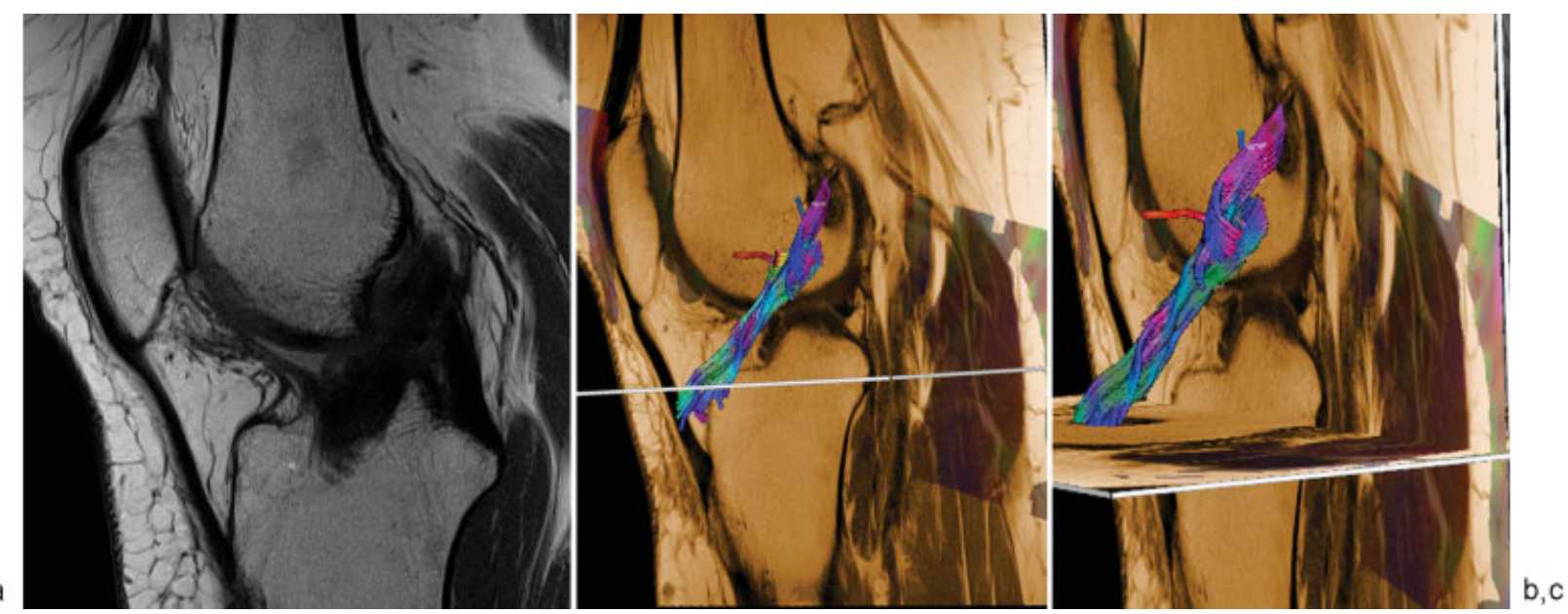

Fig. 8 Diffusion tensor imaging of anterior cruciate ligament (ACL) graft. (a) Sagittal proton-density weighted image demonstrates intact ACL graft. (b) Sagittal and (c) oblique three-dimensional views of fiber tractography showing gross morphology of the ACL graft.
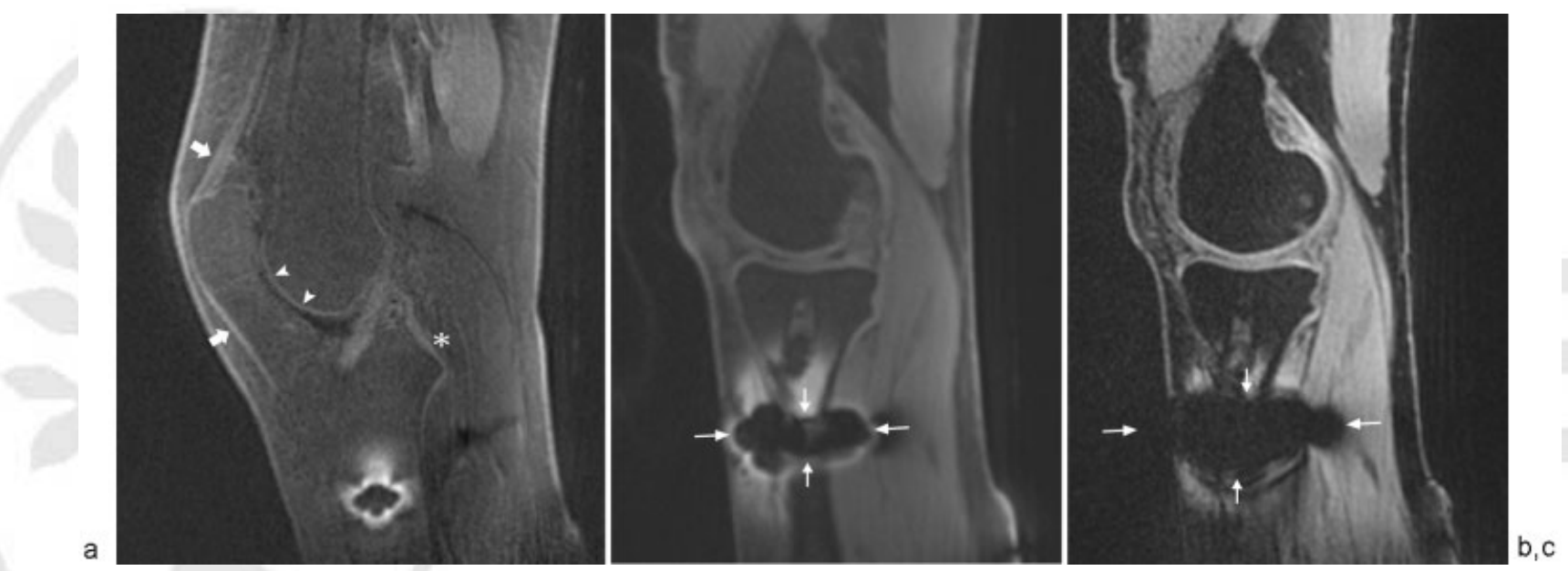

Fig. 9 Ultrashort TE (UTE) MR imaging after anterior cruciate ligament (ACL) reconstruction. (a) UTE subtraction image (same patient as Fig. 1) shows high signal intensity of the ACL graft. High-intensity linear signal near the osteochondral junction at the trochlea (arrowheads), extensor apparatus (arrows), and posterior cruciate ligament (asterisk) can also be seen. (b) Sagittal UTE images with $60 \mu \mathrm{s}$ and (c) $3.92 \mathrm{~ms}$ TEs show more pronounced dephasing artifact surrounding hardware fixation at the longer TE.

images with very short TEs, typically in the range of 8 to 250 $\mu \mathrm{s}^{36,37}$ As a result, signal can be acquired from short $\mathrm{T} 2$ structures, such as tendons and ligaments. Several UTE techniques focusing on the method of image acquisition have been developed. $^{38,39}$ The author's institution uses a dual-echo 3D UTE sequence with minimum $\mathrm{TE}=60 \mu \mathrm{s}$. Difference images are formed to suppress signals from long T2 components by subtracting the later echo time $(\mathrm{TE}=3.92 \mathrm{msec}$ ) image from the first image $(\mathrm{TE}=60 \mu \mathrm{s})$. As with conventional MR techniques, UTE can also be used for quantitative $\mathrm{T}^{*}$ mapping of tissues. ${ }^{38}$

UTE imaging provides new options to visualize anatomy and demonstrate disease of the knee. Also, contrast enhancement within the normal and injured ACL can be observed with use of UTE sequences. Finally, UTE imaging can be used to reduce susceptibility artifacts from metallic implants. ${ }^{36,37}$ This may improve visualization of the bone tunnels after ACL surgery and ultimately may lead to better assessment of bone tunnel healing (-Fig. 9).

\section{Conclusion}

1. The surgical trend toward anatomical ACL reconstruction necessitates the use of imaging studies.

2. Novel ACL repair techniques are increasingly being performed as an alternative to ACL reconstruction to improve clinical outcomes. These repair techniques have to be performed within several weeks after the rupture. This requires the prompt availability of MRI.

3. Volumetric CT is the golden standard for the evaluation of bone tunnel placements after ACL surgery.

4. The repaired or regenerated ACL may display high signal intensity on MRI, even in a clinically stable knee.

5. Advanced MR techniques can be used as an adjunct to conventional imaging to monitor the healing ACL quantitatively. UTE imaging may provide new options to visualize the postoperative ACL. 


\section{Conflict of Interest}

The first author (P.V.D.) is a consultant for and has received compensation from Soft Tissue Regeneration, Inc., New Haven, CT, USA.

\section{Acknowledgments}

We would like to thank Robert Poggie and Joseph W. Reilly, Soft Tissue Regeneration, Inc., New Haven, CT, USA, for discussions regarding ACL regeneration and for providing Fig. 4.

\section{References}

1 Steiner ME, Murray MM, Rodeo SA. Strategies to improve anterior cruciate ligament healing and graft placement. Am J Sports Med 2008;36(1):176-189

2 Kiapour AM, Murray MM. Basic science of anterior cruciate ligament injury and repair. Bone Joint Res 2014;3(2):20-31

3 Murawski CD, van Eck CF, Irrgang JJ, Tashman S, Fu FH. Operative treatment of primary anterior cruciate ligament rupture in adults. J Bone Joint Surg Am 2014;96(8):685-694

4 Murray MM, Vavken P, Fleming BC, eds. The ACL Handbook: Knee Biology, Mechanics, and Treatment. New York, NY: Springer; 2013

5 Nau T, Teuschl A. Regeneration of the anterior cruciate ligament: current strategies in tissue engineering. World J Orthod 2015;6(1): 127-136

6 Fu FH, Bennett CH, Lattermann C, Ma CB. Current trends in anterior cruciate ligament reconstruction. Part 1: Biology and biomechanics of reconstruction. Am J Sports Med 1999;27(6):821-830

7 Janssen RPA, Scheffler SU. Intra-articular remodelling of hamstring tendon grafts after anterior cruciate ligament reconstruction. Knee Surg Sports Traumatol Arthrosc 2014;22(9):2102-2108

$8 \mathrm{Kim}$ HS, Seon JK, Jo AR. Current trends in anterior cruciate ligament reconstruction. Knee Surg Relat Res 2013;25(4):165-173

9 Schindler OS. Surgery for anterior cruciate ligament deficiency: a historical perspective. Knee Surg Sports Traumatol Arthrosc 2012; 20(1):5-47

10 Chambat P, Guier C, Sonnery-Cottet B, Fayard J-M, Thaunat M. The evolution of ACL reconstruction over the last fifty years. Int Orthop 2013;37(2):181-186

11 Rayan F, Nanjayan SK, Quah C, Ramoutar D, Konan S, Haddad FS. Review of evolution of tunnel position in anterior cruciate ligament reconstruction. World J Orthod 2015;6(2):252-262

12 Eggli S, Kohlhof H, Zumstein M, et al. Dynamic intraligamentary stabilization: novel technique for preserving the ruptured ACL. Knee Surg Sports Traumatol Arthrosc 2015;23(4):1215-1221

13 Mackay GM, Blyth MJ, Anthony I, Hopper GP, Ribbans WJ. A review of ligament augmentation with the InternalBrace ${ }^{\mathrm{TM}}$ : the surgical principle is described for the lateral ankle ligament and ACL repair in particular, and a comprehensive review of other surgical applications and techniques is presented. Surg Technol Int 2015;26:239-255

14 William RW, Bertollo N, Arciero RA, Stanton RA, Poggie RA. Longterm in-vivo evaluation of a resorbable PLLA scaffold for regeneration of the ACL. Orthop J Sports Med 2015;3(2, Suppl)

15 Schliemann B, Lenschow S, Domnick C, et al. Knee joint kinematics after dynamic intraligamentary stabilization: cadaveric study on a novel anterior cruciate ligament repair technique. Knee Surg Sports Traumatol Arthrosc 2015; August 4 (Epub ahead of print)

16 Reichert M, Ai T, Morelli JN, Nittka M, Attenberger U, Runge VM. Metal artefact reduction in MRI at both 1.5 and $3.0 \mathrm{~T}$ using slice encoding for metal artefact correction and view angle tilting. $\mathrm{Br} \mathrm{J}$ Radiol 2015;88(1048):20140601
17 Kohl S, Evangelopoulos DS, Kohlhof $\mathrm{H}$, et al. Anterior crucial ligament rupture: self-healing through dynamic intraligamentary stabilization technique. Knee Surg Sports Traumatol Arthrosc 2013;21(3):599-605

18 Henle P, Röder C, Perler G, Heitkemper S, Eggli S. Dynamic Intraligamentary Stabilization (DIS) for treatment of acute anterior cruciate ligament ruptures: case series experience of the first three years. BMC Musculoskelet Disord 2015;16:27

19 Mackay GM, Anthony IC, Jenkins PJ, Blyth MJ. Anterior cruciate ligament repair revisited. Preliminary results of primary repair with internal brace ligament augmentation: a case series. Orthop Muscul Syst 2015;4:188

20 Parkar AP, Adriaensen ME, Fischer-Bredenbeck C, et al. Measurements of tunnel placements after anterior cruciate ligament reconstruction - A comparison between $\mathrm{CT}$, radiographs and MRI. Knee 2015;22(6):574-579

21 Purnell ML, Larson AI, Clancy W. Anterior cruciate ligament insertions on the tibia and femur and their relationships to critical bony landmarks using high-resolution volume-rendering computed tomography. Am J Sports Med 2008;36(11):2083-2090

22 Bernard M, Hertel P, Hornung H, Cierpinski T. Femoral insertion of the ACL. Radiographic quadrant method. Am J Knee Surg 1997; 10(1):14-21; discussion 21-22

23 Parkar AP, Adriaensen MEAMP, Strand T, Inderhaug E, Harlem T, Solheim E. How to read post-operative radiographs and CT scans after single-bundle anterior cruciate ligament reconstruction. Skeletal Radiol 2013;42(11):1489-1500

24 Parkinson B, Gogna R, Robb C, Thompson P, Spalding T. Anatomic $A C L$ reconstruction: the normal central tibial footprint position and a standardised technique for measuring tibial tunnel location on 3D CT. Knee Surg Sports Traumatol Arthrosc 2015

25 Groves C, Chandramohan M, Chew C, Subedi N. Use of CT in the management of anterior cruciate ligament revision surgery. Clin Radiol 2013;68(10):e552-e559

26 Murakami Y, Sumen Y, Ochi M, Fujimoto E, Deie M, Ikuta Y. Appearance of anterior cruciate ligament autografts in their tibial bone tunnels on oblique axial MRI. Magn Reson Imaging 1999; 17(5):679-687

27 Meyers AB, Haims AH, Menn K, Moukaddam H. Imaging of anterior cruciate ligament repair and its complications. AJR Am J Roentgenol 2010;194(2):476-484

28 Howell SM, Knox KE, Farley TE, Taylor MA. Revascularization of a human anterior cruciate ligament graft during the first two years of implantation. Am J Sports Med 1995;23(1):42-49

29 Waltz RA, Solomon DJ, Provencher MT. A radiographic assessment of failed anterior cruciate ligament reconstruction. Can magnetic resonance imaging predict graft integrity? Am J Sports Med 2014; 42(7):1652-1660

30 Van Dyck P, Gielen JL, Vanhoenacker FM, Wouters K, Dossche L, Parizel PM. Stable or unstable tear of the anterior cruciate ligament of the knee: an MR diagnosis? Skeletal Radiol 2012;41(3):273-280

31 Fleming BC, Vajapeyam S, Connolly SA, Magarian EM, Murray MM. The use of magnetic resonance imaging to predict ACL graft structural properties. J Biomech 2011;44(16):2843-2846

32 Biercevicz AM, Akelman MR, Fadale PD, et al. MRI volume and signal intensity of ACL graft predict clinical, functional, and patient-oriented outcome measures after ACL reconstruction. Am J Sports Med 2015;43(3):693-699

33 Biercevicz AM, Murray MM, Walsh EG, Miranda DL, Machan JT, Fleming $\mathrm{BC}$. T2 * MR relaxometry and ligament volume are associated with the structural properties of the healing ACL. J Orthop Res 2014;32(4):492-499

34 Yang X, Li M, Chen D, et al. Diffusion tensor imaging for anatomical and quantitative evaluation of the anterior cruciate ligament and ACL grafts: a preliminary study. J Comput Assist Tomogr 2014; 38(4):489-494

35 Yang X, Chen D, Li M, Shi D, Zhu B, Jiang Q. Diffusion tensor imaging of the anterior cruciate ligament graft after reconstruction: 
repeatability and diffusion tensor imaging metrics. J Comput Assist Tomogr 2015;39(2):244-249

36 Gatehouse PD, Bydder GM. Magnetic resonance imaging of short T2 components in tissue. Clin Radiol 2003;58(1):1-19

37 Gatehouse PD, Thomas RW, Robson MD, Hamilton G, Herlihy AH, Bydder GM. Magnetic resonance imaging of the knee with ultrashort TE pulse sequences. Magn Reson Imaging 2004;22(8):1061-1067
38 Du J, Carl M, Bae WC, et al. Dual inversion recovery ultrashort echo time (DIR-UTE) imaging and quantification of the zone of calcified cartilage (ZCC). Osteoarthritis Cartilage 2013;21(1): 77-85

39 Grodzki DM, Jakob PM, Heismann B. Ultrashort echo time imaging using pointwise encoding time reduction with radial acquisition (PETRA). Magn Reson Med 2012;67(2):510-518 